\title{
Trabajo voluntario para disminución de listas de espera de Cirugía Plástica en hospitales núblicos de Chile. Operativo Hospital de Valdivia, enero 2019
}

\section{Volunteer work to decrease Plastic Surgery waiting lists in public hospitals in Chile. Medical mission Valdivia Hospital, Janury 2019}

Claudio THOMAS BAS*, Carlos FONFACH ZAHN**

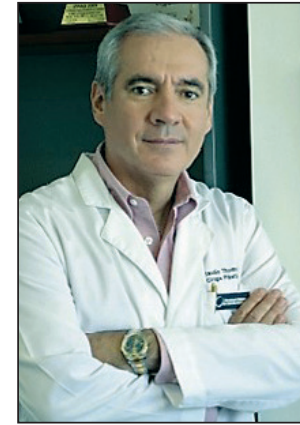

Thomas Bas C.

\section{Resumen}

Introducción y Objetivo. Algunas intervenciones de Cirugía Plástica, por ser menos prioritarias en un sistema con carencias económicas propias de un país en vías de desarrollo como es Chile, tienen una larga lista de espera en los hospitales públicos. Con un objetivo de servicio público, miembros de la Sociedad Chilena de Cirugía Plástica desarrollan desde hace 2 años un operativo altruista de salud en colaboración con el Ministerio de Salud de Chile.

Presentamos en este trabajo la última campaña de cirugía de reducción de mama realizada en 2019.

Material y método. Revisión retrospectiva del operativo de salud para cirugía de reducción mamaria en el Hospital de Valdivia (Chile). Mostramos el protocolo de selección de pacientes, las técnicas quirúrgicas utilizadas y la evaluación de resultados.

Resultados. A lo largo de 2 días fueron intervenidas 15 pacientes por un grupo de 8 especialistas divididos en 2 grupos de trabajo. Las complicaciones, todas ellas mediatas, fueron menores.

Conclusiones. Si bien estos operativos quirúrgicos no son la solución para las listas de espera de Cirugía Plástica en los hospitales públicos de Chile, ayudan a su mejoría y demuestran ser efectivos con una planificación científica y administrativa adecuada.
Palabras clave Reducción mamaria, Cirugía Plástica, Misiones sanitarias.

\section{Nivel de evidencia científica Recibido [esta versión] Aceptado}

5 Estudios económicos

$21 \mathrm{mayo} / 2019$

3 junio/2019
Abstract

Background and Objective. Some Plastic Surgery operations have a long wait in public hospitals in Chile as they are less priority in a system with the typical economic deficiencies of a developing country. With a public service objective, members of the Chilean Society of Plastic Surgery have been developing an altruistic health operation in the last 2 years, in collaboration with the Ministry of Health.

We present the latest campaign of breast reduction surgery performed in 2019 .

Methods. Retrospective review of the health operative for breast reduction surgery at the Valdivia Hospital (Chile). We present the patient selection protocol, the surgical techniques used and the evaluation of results.

Results. Throughout 2 days, 15 patients were operated on by a group of 8 specialists divided into 2 work groups. All the complications were mediate and minor.

Conclusions. Although these surgical operatives are not the solution for the waiting lists of Plastic Surgery in public hospitals in Chile, they help to improve them and prove to be effective with adequate scientific and administrative planning.

$\begin{array}{lr}\begin{array}{l}\text { Key words } \\ \text { Breast reduction, Plastic Surgery, } \\ \text { Medical missions. }\end{array} \\ \text { Level of evidence } & 5 \text { Economic studies } \\ \text { Received [this version] } & 21 \text { May/2019 } \\ \text { Accepted } & 3 \text { June / } 2019\end{array}$

Conflicto de intereses: Los autores declaran no tener ningún interés financiero relacionado con el contenido de este artículo. Financiación: No hubo fuentes externas de financiación para este trabajo. 
Introducción

En los hospitales públicos de Chile existen listas de espera de Cirugía Plástica de numerosos pacientes; hasta 4366 contabilizados en agosto de 2018 (1) $^{(1)}$ Esto guarda relación con la falta de especialistas ${ }^{(2)}$ existente en el sistema público de salud chileno y la condición administrativa de las enfermedades sin "garantías explícitas en salud" (GES), es decir, aquellas que no tienen garantía de oportunidad y acceso. Las patologías no GES de Cirugía Plástica tienen un tiempo de espera muy prolongado en nuestro país, por ser consideradas menos prioritarias en un sistema con las carencias de recursos económicos propias de un país en vías de desarrollo.

Por este motivo y con un objetivo de servicio público, miembros de la Sociedad Chilena de Cirugía Plástica (SCCP) participan desde hace 2 años de forma voluntaria en operativos de salud organizados por la Secretaría de Redes Asistenciales del Ministerio de Salud de Chile, junto con colegas de otras especialidades.

En el presente artículo mostramos las características y los resultados del operativo quirúrgico efectuado en la ciudad de Valdivia, ubicada a unos $850 \mathrm{~km}$. aproximadamente al sur de Santiago.

Valdivia es la capital de la Región de los Ríos. Su hospital base cubre a una población de 350.000 habitantes aproximadamente y cuenta con 1 solo cirujano plástico. La lista de espera más numerosa de Cirugía Plástica en este hospital es la de reducción mamaria, motivo por el cual se decide efectuar un operativo para disminuirla.

Material y Método

Seleccionamos a las pacientes para reducción mamaria con los siguientes criterios de inclusión: 1) volumen

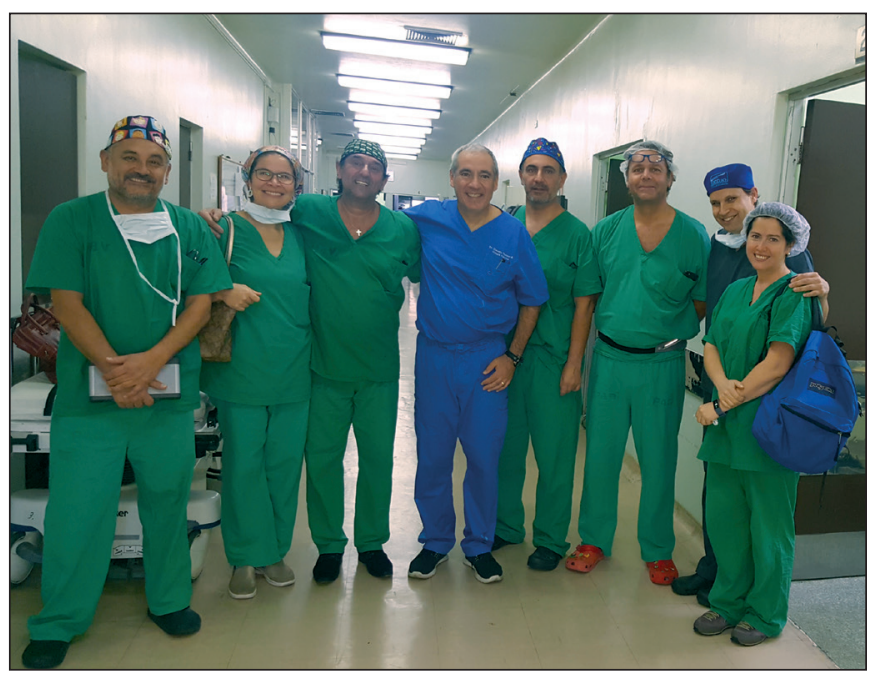

Figura 1. Imagen del grupo de trabajo al final de una de las largas sesiones de trabajo. De izquierda a derecha los doctores. Rivera, Rozas, Casthilo, Thomas, Cuadra, Shultz, Fonfach y Albornoz. aproximado de reducción mayor de 350 gr. por mama. 2) Índice de masa corporal (IMC) inferior a $30 \mathrm{~kg} . / \mathrm{m}^{2} .3$ ) No fumadoras. 4) Menores de 60 años de edad o mayores de 60 sin enfermedades asociadas.

Esta selección fue desarrollada por el cirujano plástico del hospital de Valdivia y determinó que 15 pacientes de la lista de espera cumplían con los requisitos de entre un total de 54 pacientes.

La edad promedio de las pacientes del grupo seleccionado fue de 39 años (máxima de 56 y mínima de 22). Todas ellas completaron un estudio preoperatorio que incluyó mamografía y ecotomografía mamaria, pruebas de coagulación, hemograma, perfil bioquímico, análisis de orina y electrocardiograma, firmaron un consentimiento informado y a todas se les tomaron fotografías clínicas antes de la intervención.

Las 15 pacientes fueron intervenidas quirúrgicamente por un grupo formado por 8 especialistas y 2 médicos en formación de subespecialidad quirúrgica, distribuidos en equipos de 4 cirujanos por paciente, en quirófanos del hospital de Valdivia, en operaciones continuas llevadas a cabo los días 18 y 19 de enero de 2019 con jornadas de 12 y 10 horas de trabajo respectivamente (Fig. 1 y 2).

Todas las pacientes fueron intervenidas bajo anestesia general, y recibieron profilaxis antibiótica y antitrombótica.

Seleccionamos las técnicas quirúrgicas empleadas según la experiencia del equipo quirúrgico. En total se emplearon las de Pitanguy, Planas y pedículo suúpero-medial, siempre simétricas.

Enviamos todos los tejidos resecados para estudio anatomopatológico con biopsia diferida y consignamos los tiempos quirúrgicos, el volumen total resecado por paciente, las complicaciones inmediatas y mediatas.

Finalmente, evaluamos el grado de satisfacción inicial de las pacientes y de los médicos participantes.

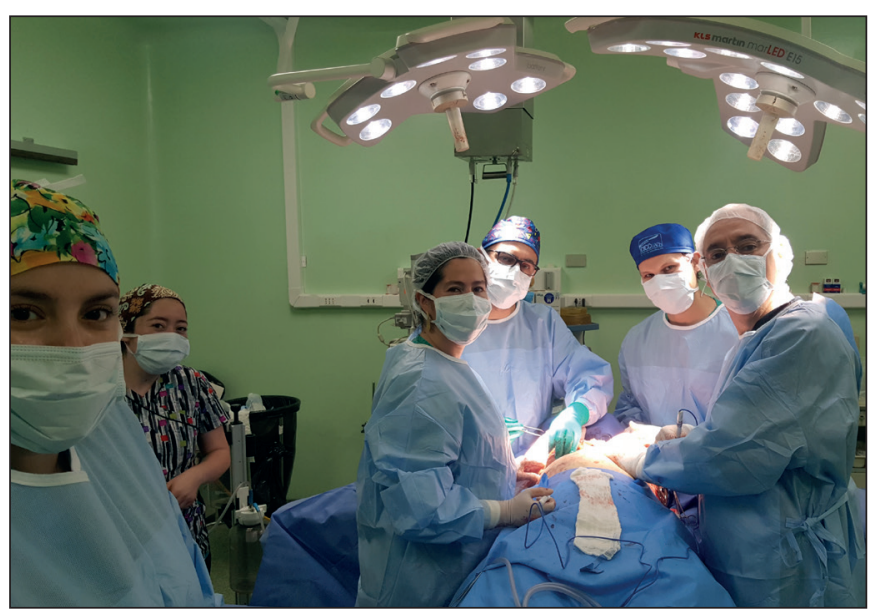

Figura 2. Uno de los equipos de cuatro cirujanos para cirugía simultánea de ambas mamas. De izquierda a derecha, los doctores Albornoz, Cuadra, Fonfach y Thomas. 
El promedio de tiempo quirúrgico empleado fue de 126 minutos por paciente (máximo de 190 y mínimo de 85).

El peso promedio de tejido mamario resecado fue de 1275 gr. (máximo de 2080 y mínimo de 690gr.).

No registramos complicaciones inmediatas, entendidas como tales las aparecidas en las primeras 24 horas de postoperatorio. Como complicaciones mediatas, aparecidas entre los 2 y los 15 días de postoperatorio contabilizamos las siguientes: 2 casos de dehiscencia parcial de heridas operatorias; 2 casos de infección menor que requirieron solo tratamiento médico; y 1 caso de epidermólisis parcial de areola.

En la evaluación de satisfacción por parte de las pacientes intervenidas 7 respondieron estar muy satisfechas, 1 satisfecha, y otras 7 están aún en control postoperatorio. Todos los médicos participantes respondieron estar muy satisfechos con la labor desarrollada.

\section{Discusión}

La reducción mamaria es una intervención de Cirugía Plástica que requiere un equipo médico entrenado y con experiencia, lo que en nuestro caso se refleja en el bajo número de complicaciones sufridas en esta serie. ${ }^{(3)}$ También es importante la formación de equipos que permitan lograr tiempos quirúrgicos cortos, que contribuyen a incrementar la seguridad de las pacientes. En el mismo sentido es muy importante una selección adecuada de los casos considerando factores de riesgo como la obesidad y el tabaquismo, ${ }^{(4)}$ en especial en este tipo de operativos quirúrgicos donde solo un especialista asume la responsabilidad y se ocupa de los tratamientos postoperatorios. En nuestros casos, solo fueron precisos controles rutinarios de seguimiento y algún tratamiento médico para resolver las complicaciones que fueron todas de poca importancia.

A la hora de valorar el grado de satisfacción por parte de las pacientes, hemos de tener en cuenta que no todas han podido valorar hasta la fecha la encuesta realizada ya que viven en zonas rurales alejadas de Valdivia y serán controladas en los próximos meses, si bien consideramos que la tendencia es a una calificación mayoritaria de conformidad con el resultado que concuerda con otras publicaciones de este mismo tipo en pacientes sometidas a reducción mamaria. ${ }^{(5)}$

En cuanto a los médicos participantes en el operativo, valoramos también el grado de satisfacción que manifestaron, probablemente relacionado con el espíritu de trabajo en equipo y el agradecimiento expresado por las pacientes en el entorno quirúrgico. ${ }^{(6)}$
Teniendo en cuenta que este tipo de operativos quirúrgicos no es la solución para las listas de espera de Cirugía Plástica en los hospitales públicos de Chile, si consideramos que son un alivio parcial para quienes esperan durante años una cirugía de este tipo, a la vez que demuestran que es posible lograr llevar a cabo procedimientos seguros cuando se hace una planificación adecuada, tanto científica como administrativa.

Para los médicos que desarrollan estas misiones de trabajo voluntario entregando conocimiento y tiempo, sin compromiso ni interés, la experiencia es muy favorable y enriquecedora del alma médica.

\section{Agradecimiento}

Queremos dar un gran agradecimiento a nuestros colegas los doctores: Claudia Albornoz, Cecilia Rozas, Antonio Casthilo, Álvaro Cuadra, Juan Rivera, Carlos Schultz, Ignacio Cifuentes y Diego Quispe, por su labor intensa y desinteresada. Sin su trabajo voluntario no hubiésemos podido efectuar este operativo de salud.

\section{Dirección del autor}

Dr. Claudio Thomas Bas

Av. Raúl Labbé 12613 of. 108.

Lo Barnechea

Santiago, Chile

Correo electrónico: claudiothomas72@gmail.com

\section{Bibliografía}

1. Oficio ordinario C2 \#3693: Información relativa a listas de espera no GES. Subsecretaria de Redes Asistenciales. Ministerio de Salud de Chile.

2. Estudio sobre determinación de brecha de médicos generales y especialistas según metodología de tasas de uso de prestaciones médicas y especializadas en Chile. Ministerio de Salud de chile. Subsecretaría de Redes Asistenciales, 13 de Diciembre de 2017.

3. Fischer JP., Wes AM., Kovach SJ. The impact of surgical resident participation in breast reduction surgery. Outcome analysis from the 2005-2011 ACS-NSQIP data sets. J Plast Surg Hand Surg. 2014; 48(5):315-21.

4. Nelson JA., Fischer JP., Chung CU., West A., Tuggle CT., Serletti JM., Kovach SJ. Obesity and early complications following reduction mammaplasty: ananalysis of 4545 patients from the 2005-2011 NSQIP data sets.J Plast Surg Hand Surg. 2014 Oct;48(5):334-339.

5. Cuevas P., Calderón ME., Erazo C., Benítez S., Andrades P. et al. Mamoplastia de reducción: resultados desde la perspectiva del paciente. Validación lingüística y psicométrica del Breast Q Reduction and Mastopexy Module Instrument. Rev.Chil.Cir. 2013; 65(2): 146.149.

6. Villafuerte-Vélez, CK. PROTG-Programa social de tratamiento de la gigantomastia, Bahía-Brasil. Cir. plást. iberolatinoam., 2018;.44 (3):341-346. 
